\title{
Potencial de Fibras Lignocelulósicas para a Produção de Etanol de Segunda Geração
}

\author{
J osé Mariano da Silva Neto; \\ Adalício Uzeda Antunes Júnior; \\ Fernanda Siqueira Lima; \\ Deborah Almeida dos Anjos
}

\begin{abstract}
Resumo:
A produção de produtos químicos e combustíveis a partir de materiais lignocelulósicos tem recebido um particular interesse de pesquisadores devido ao impacto negativo dos combustíveis fósseis no meio ambiente, como também a crescente preocupação com as questões geopolíticas do petróleo em todo o mundo. As pesquisas atuais mostram como tecnologia alternativa a produção de etanol de segunda geração a partir de materiais lignocelulósicos. O etanol de segunda geração corresponde a produção de etanol, entretanto, utilizando-se como matéria-prima materiais lignocelulósicos. Os materiais lignocelulósicos são compostos basicamente de celulose, hemicelulose e lignina. Esses três componentes são determinantes para a produção desse biocombustível, uma vez que a quebra dessas moléculas irá produzir açúcares que podem com o auxílio de leveduras ser transformados bioquimicamente em etanol e outros produtos químicos. A região do semiárido nordestino, compreendido entre oito estados nordestinos: Alagoas, Bahia, Ceará, Paraíba, Pernambuco, Piauí, Rio Grande do Norte e Sergipe possui materiais promissores para a produção desses biocombustíveis como o bagaço e palha da cana-de-açúcar, fibra do sisal, palma forrageira e bagaço do pedúnculo do caju. Além da escolha adequada da matéria-prima para a produção do etanol 2G, as etapas que envolvem esse processo, pré-tratamento, hidrólise, fermentação e destilação, irão depender primordialmente da cultura escolhida e sua composição físico-química. Com isso o objetivo principal desse trabalho é mostrar que o potencial de fibras lignocelulósicas para a produção de etanol de segunda geração e estudar três tipos principais de pré-tratamento para as mesmas: ácido, básico e uma combinação ácido-base.
\end{abstract}

\section{Palavras-chave:}

Pré-tratamento; Material Lignocelulósico; Açúcares Fermentescíveis; Nordeste 


\section{Energia Renovável no Brasil}

Energia renovável é, por definição, sustentável e limpa e, além disso, oferece a oportunidade de enfrentar a crescente diminuição do uso de recursos fósseis e os impactos a eles associados (CHEN et al., 2010). Globalmente, o Brasil está numa posição favorável quando se fala em fontes renováveis de energia. Em 2013 mais de $40 \%$ de toda a energia primária produzida no Brasil veio de fontes renováveis de energia (EPE, 2014), um valor relativamente alto quando comparado com a média mundial que foi aproximadamente $13 \%$ (IEA,2013).

A maioria das fontes renováveis usadas no país provém de produtos da cana-de-açúcar (16,1\%), energia hidrelétrica (15,5\%) e outras biomassas (8,3\%). Eólica, solar e outras fontes renováveis ainda são pouco difundidas, representando menos de $5 \%$ do total de energia primária produzida no Brasil (EPE,2014).

No Brasil, é esperado nos próximos 10 anos um aumento de 5,3\% na demanda anual energética, alcançando 372 milhões de TEP (tonelada equivalente de petróleo) até 2020 (TOLMASQUIM, 2012). Mesmo com a grande diversificação da matriz energética brasileira, o petróleo ainda é a principal fonte energética do país, com uma quota de 37,8\% (FERREIRA, 2007).

Por esse fato, pesquisas têm sido desenvolvidas para que o uso desse combustível fóssil possa ser diminuído, por este apresentar questões delicadas na geopolítica mundial e também devido a grande preocupação ambiental e as emissões de poluentes atmosféricos através da utilização desse tipo de combustível.

De acordo com The Economist (2009), a economia brasileira deverá se expandir do nono para a quinta maior do mundo em 2025, e sabendo que o país possui um grande potencial em termos de recursos naturais e da expansão de uma agroindústria, a produção de biocombustíveis, a partir de pontos de vista econômico, ambiental e social, é vista como uma alternativa atraente para a produção de energia renovável no Brasil.

No Brasil, para a produção do etanol, principal combustível produzido no país, a cana-de-açúcar é atualmente a principal matéria-prima utilizada, sendo 1 tonelada de cana suficiente para produzir 85 litros de álcool (RODRIGUES,2010), tendo hoje o etanol mais eficiente do mundo, correspondente a $18 \%$ da matriz energética do país, com produção anual de 26 bilhões de litros. Segundo informações do ex-ministro de Minas e Energia, Márcio Zimmermann, estimativas apontam que no ano de 2019 a produção de etanol deverá alcançar 64 bilhões de litros anuais, mais que o dobro da produção atual.

Nesse cenário, o Brasil tem o potencial para expandir a sua produção de etanol no futuro (CHUN et al., 2011). Esse potencial inclui a expansão da área de cultivo, melhoramento na área da agricultura também a introdução de novas vias de processamento industrial (LEITE, 2009). Nessas novas vias de processamento industrial podem-se incluir o melhoramento do etanol de primeira geração (fermentação de açúcares) como também de segunda geração (conversão da biomassa lignocelulósica).

O processamento industrial de segunda geração poderá melhorar fortemente a produção de etanol durante todo o ano, como o abastecimento de culturas lignocelulósicas é menos sazonal do que a cana-de-açúcar. Apesar da investigação em curso em tecnologia de segunda geração, o desempenho econômico de desenvolvimentos tecnológicos futuros permanece incerto (CHOVAU, 2013).

Ao lado de cana, outras matérias-primas de biomassa têm sido propostas para a produção de etanol devido ao seu potencial de rendimento, composição ou a tolerância ao clima e / ou características do solo em outras áreas de cultivo (BNDS, 2008).

\section{Fibras Lignocelulósicas}

As matérias-primas lignocelulósicas são as fontes renováveis mais abundantemente encontradas na natureza, sendo compreendidas, majoritariamente, pelos materiais agroindustriais, pelos resíduos urbanos e pelas madeiras de angiospermas e gimnospermas (CASTRO et al., 2010).

A lignocelulose é um complexo rígido composto de diferentes moléculas, que constitui quase a totalidade da parcela estrutural das plantas, ou seja, a maior porcentagem da biomassa dos vegetais. É um substrato de custo baixo que é considerado resíduo final industrial e agrícola, e as composições desses materiais variam e são constituídas principalmente por celulose (35-50\%) seguida por 
hemicelulose (20-30\%) e lignina (10-25\%) e podem ser transformadas em energia e produtos químicos (KUMAR et al., 2009)

A celulose de fórmula $\left(\mathrm{C}_{6} \mathrm{H}_{10} \mathrm{O}_{5}\right)_{n}$ é um polissacarídeo C6 formado por uma longa cadeia de moléculas de glicose. A hemicelulose de fórmula $\left(\mathrm{C}_{5} \mathrm{H}_{8} \mathrm{O}_{4}\right)_{n}$ e $\left(\mathrm{C}_{6} \mathrm{H}_{10} \mathrm{O}_{5}\right)$ é um componente relativamente amorfo, que é mais facilmente quebrada quimicamente por calor do que a celulose é constituída por uma mistura de polissacarídeos C6 (galactose e manose) e polissacarídeos C5 (xilose e arabinose). A lignina é essencialmente o cimento que propicia a rigidez estrutural das plantas e árvores, formada por uma rede polimérica tridimensional de unidades metoxilas, arilpropanos e hidroxifenóis. A fórmula empírica deste polímero complexo é $\mathrm{C}_{9} \mathrm{H}_{10} \mathrm{O}_{2}\left(\mathrm{OCH}_{3}\right)_{\mathrm{n}}$ no qual n é a razão de $\mathrm{CH}_{3} \mathrm{O}$ para grupos C9:n = 1,4; 0,94 e 1,18 para as madeiras duras, moles e gramíneas, respectivamente. O que dá a rigidez a esta rede polimérica são as ligações cruzadas. A lignina é o maior constituinte não carboidrato, correspondendo entre 15 e $25 \%$ do vegetal (RODRIGUES, 2011)

A composição básica da biomassa lignocelulósica depende do vegetal de origem, conforme Tabela 1, e, no caso de biomassa agroflorestais, da região, idade e período de coleta do material. Segundo Lima \& Rodrigues (2007), a escolha da espécie produtora de biomassa é um passo fundamental na produção do etanol celulósico. Deve ser levada em conta sua produtividade, a produtividade de celuloses e hemiceluloses e a produtividade e tipo de lignina presente.

Tabela 1 - Composição química de biomassa lignocelulósica

\begin{tabular}{cccc}
\hline Biomassa & \% Celulose & \% Hemicelulose & \% Lignina \\
Lignocelulósica & $40-44$ & $30-32$ & $22-25$ \\
Palha de cana & $32-48$ & $19-24$ & $23-32$ \\
Bagaço de cana & 30 & 50 & 15 \\
Palha de trigo & 73,1 & 14,2 & 11 \\
Sisal & $38-40$ & 28 & $7-21$ \\
Forragem de milho & 45 & 35 & 15 \\
Espiga de milho & $31-45$ & $27-38$ & $14-19$ \\
Palha de cevada & $36-43$ & $0,15-0,25$ & $41-45$ \\
Fibra de côco & & &
\end{tabular}

Fonte: Gomez et al., (2010)

\subsection{Celulose}

A celulose, principal componente da parede celular da fibra vegetal, é um polímero linear que contém até 15.000 unidades de $\beta$-D-glicoses unidas por ligações glicosídicas $\beta$-1,4 carbono-carbono e por ligações de hidrogênio intramoleculares e intermoleculares, conforme Figura 1. Segundo Yang et al. (2010) é a matéria orgânica mais abundante sobre a Terra, consistindo em cerca de 50\% de toda a biomassa e uma produção anual de aproximadamente 100 bilhões de toneladas.

Figura 1 - Estrutura química da celulose

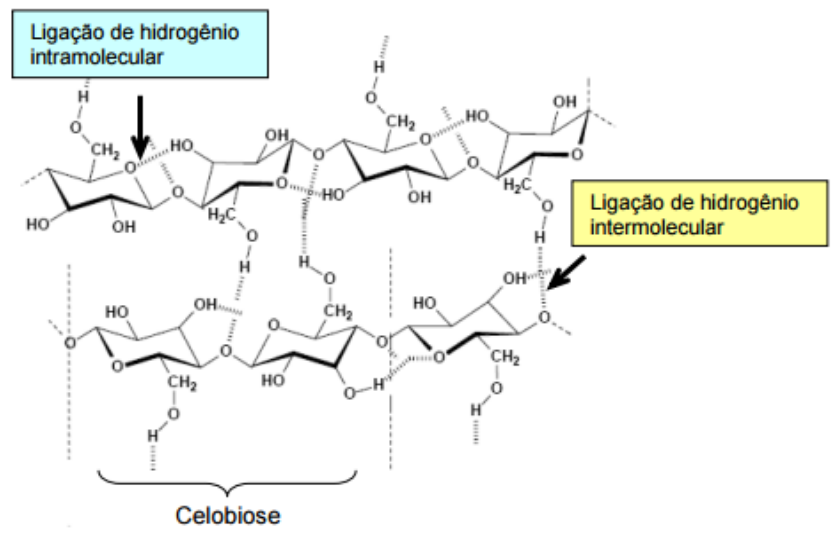

Fonte: Morais, 2005 
As ligações intermoleculares são responsáveis pela rigidez; e as ligações intramoleculares são responsáveis pela formação de fibrilas, estruturas altamente ordenadas que se associam formando as fibras de celulose. Estas características, juntamente com o envoltório de lignina, conferem à macromolécula celulose grande resistência à hidrólise, o que representa um grande desafio para a utilização dos materiais lignocelulósicos em aplicações biotecnológicas, como a produção de etanol de segunda geração (ARANTES \& SADDLER, 2010).

\subsection{Hemicelulose}

As hemiceluloses são outros tipos de componentes essenciais presentes na parede celular das plantas e estão intimamente ligadas à celulose. São polímeros de açúcar que constitui normalmente $20-40 \%$ em peso da biomassa vegetal, cuja composição pode aparecer condensada em proporções variadas. Diferentemente da celulose, as hemiceluloses não são quimicamente homogêneas, apresenta massa molar baixa e não contém regiões cristalinas, sendo assim mais suscetível à hidrólise sob condições mais amenas e variam em suas estruturas e nas composições dependendo da fonte natural (HUBER et al. 2006).

A Tabela 2 resume as principais diferenças entre a celulose e hemicelulose.

Tabela 2 - Diferenças entre celulose e hemicelulose

\begin{tabular}{cc}
\hline Celulose & Hemicelulose \\
\hline Unidades de glicose unidas entre si & Unidades de diferentes pentoses e \\
hexoses ligadas entre si \\
Alto grau de polimerização (1000 a 15000 & Baixo grau de polimerização \\
unidades de glicose) & (60 a 300 unidades de açúcares) \\
Forma arranjo fibroso & Não forma arranjo fibroso \\
Apresenta regiões amorfas e cristalinas & Apresenta somente regiões amorfas \\
É atacada lentamente por ácido & É atacada rapidamente por ácido \\
inorgânico diluído a quente & inorgânico diluído a quente \\
É insolúvel em álcalis & É solúvel em álcalis \\
\hline
\end{tabular}

Fonte: PEREIRA Jr. et al. (2008)

\subsection{Lignina}

É constituída por polímero complexo de estrutura amorfa, com componentes aromáticos e alifáticos, que se associa a celulose e hemicelulos e durante a formação da parede celular dos vegetais e tem como finalidade conferir rigidez à mesma. Sua concentração nas fibras influencia a estrutura, as propriedades, a morfologia, a flexibilidade e a taxa de hidrólise (CARDOSO, 2008; SILVEIRA, 2008).

A lignina é uma macromolécula complexa, formada pela polimerização radicalar de unidades fenil-propano, que são três alcoóis (Figura 3) aromáticos: p-cumarilico, p-coniferílico e p-sinapílico (MELO, 2010; CARDOSO, 2008). 
Figura 3 - Estruturas moleculares precursoras da lignina: (a) Álcool p-sinapílico, (b) Álcool pconiferílico e (c) Álcool p-cumarílico<smiles>COc1cc(C=CC(=O)O)cc(OC)c1O</smiles>

(a)<smiles>COc1cc(C=CC=O)ccc1O</smiles>

(b)<smiles>OCC=Cc1ccc(O)cc1</smiles>

(c)

Fonte: MELO, 2010

A degradação da lignina apresenta grande importância econômica, tornando disponíveis substâncias de interesse na indústria, pecuária e na agricultura. A lignina pode ainda ser utilizada na produção de resinas fenólicas ou podem sofrer gaseificação com oxigênio, fornecendo gás de síntese, que é essencial na produção de metanol e pode ser utilizado como importante composto na produção de uma ampla variedade de produtos químicos (SCHUCHARDT \& RIBEIRO, 2001).

\section{Pré-Tratamentos de Materiais Lignocelulósicos}

Materiais lignocelulósicos são compostos principalmente por celulose, hemicelulose e lignina em uma estrutura complexa, na qual é resistente à degradação. Os problemas enfrentados na etapa de prétratamento de conversão de biomassa em açúcares fermentescíveis são justificados devido à baixa acessibilidade à celulose devido à rígida associação desta com a lignina, conforme mostrado na Figura 4 (GUPTA et al., 2010). Em outras palavras, o pré-tratamento é um processo unitário crucial e dispendioso na conversão de materiais lignocelulósicos em biocombustíveis.

Figura 4-Estruturas de materiais lignocelulósicos antes e após o pré-tratamento

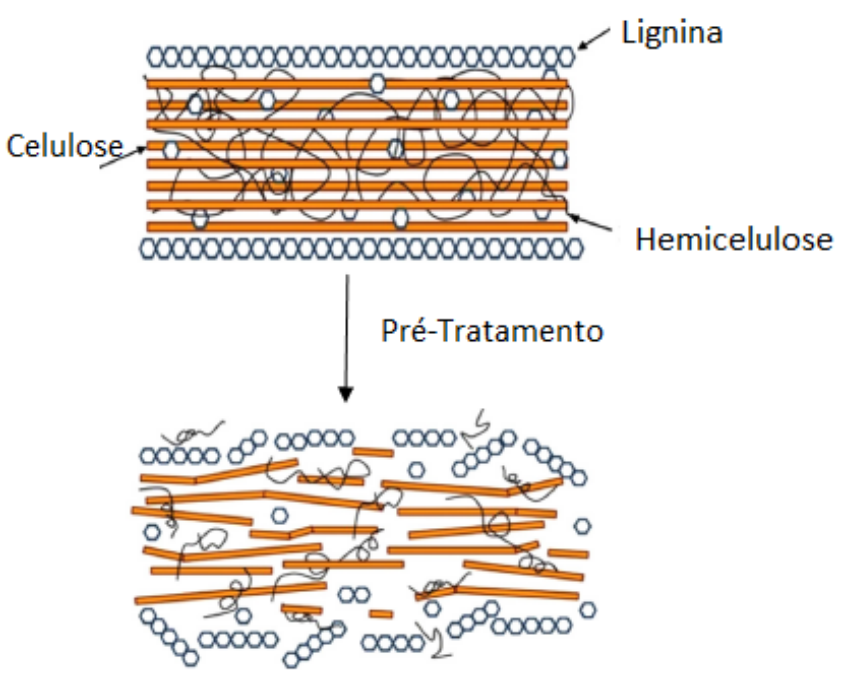

Fonte: MOOD et al., 2013

Um procedimento adequado de pré-tratamento envolve a quebra de ligação de hidrogênio na celulose cristalina, a decomposição das hemiceluloses e lignina, e finalmente, o aumento da porosidade e área superficial da celulose para a subsequente etapa (LI et al., 2010). Existem diversos métodos de pré-tratamento incluindo, tratamentos físicos (trituração e moagem, micro-ondas e extrusão), pré- 
tratamentos químicos (alcalino, ácido, organosolv, ozonólise e líquidos iônicos), físico-químicos (explosão a vapor, hidrotérmico, explosão de fibra com amônia, oxidação úmida e explosão de $\mathrm{CO}_{2}$ ) e tratamentos biológicos.

\subsection{Pré-tratamento ácido}

Pré-tratamentos ácidos, especificamente, os que utilizam ácido sulfúrico como catalisador, são os mais comumente empregados para materiais lignocelulósicos em que polissacarídeos, especialmente hemiceluloses, são hidrolisados em monossacarídeos facilitando a acessibilidade da molécula de celulose.

Esse tipo de pré-tratamento pode ser realizado utilizando baixas concentrações de ácido e elevadas temperaturas ou altas concentrações de ácido e baixas temperaturas (TAHERZADEH et al., 2008). Industrialmente, ácidos diluídos são mais atrativos por baixas concentrações de inibidores da fermentação serem geradas durante essa etapa do processo.

Pinheiro et al. (2011) avaliou o pré-tratamento termoquímico do bagaço da cana-de-açúcar para a produção de açúcares fermentescíveis e usou as seguintes condições para obter uma eficiência de hidrólise de 57,40\%: temperatura de $120^{\circ} \mathrm{C}$, concentração de ácido sulfúrico de $0,5 \mathrm{M}$ e tempo de reação de 1 minuto.

Campos et al. (2013) estudou o pré-tratamento ácido da cana-de-açúcar com $\mathrm{H}_{2} \mathrm{SO}_{4}$ a uma concentração de $0,75 \%(\mathrm{v} / \mathrm{v})$, temperatura de $120^{\circ} \mathrm{C}$ durante 45 minutos, nessas condições foi obtido o mais eficiente processo na solubilização da hemicelulose e preservação da estrutura.

\subsection{Pré-tratamento alcalino}

Esse tipo de pré-tratamento tem como principais funções remover lignina, grupos acetil e diferentes ácidos urônicos que podem inibir a acessibilidade à celulose para a posterior sacarificação (LI et al., 2010).

A solubilização de hemiceluloses e celulose nesse método é menor que em processos ácidos e hidrotérmicos (CAVALHEIRO et al., 2008)

De acordo com Mosier et al. (2009), o pré-tratamento alcalino pode ser realizado em condições ambientes, com a ressalva, de que esse processo exigirá um tempo superior ao convencional quando se utilizam pressão e temperatura.

Hidróxido de sódio $(\mathrm{NaOH})$, hidróxido de potássio $(\mathrm{KOH})$, hidróxido de cálcio $(\mathrm{CaOH})$ e amônia $\left(\mathrm{NH}_{3}\right)$ são os reagentes mais comumente empregados nesse tipo de pré-tratamento. Oliveira et al. (2010) estudou o pré-tratamento alcalino utilizando $\mathrm{NaOH}(1 \%)$ a $100^{\circ} \mathrm{C}$ por $60 \mathrm{~min}$, resultou em 80,0\% de deslignificação da palha da cana-de-açúcar.

Nascimento (2011) trabalhando com bagaço de cana-de-açúcar com $\mathrm{NaOH}$ a 7\% por $30 \mathrm{~min}$ a $120^{\circ} \mathrm{C}$, obteve a deslignificação do material em $95 \%$ de biomassa.

\subsection{Combinação dos pré-tratamentos ácido e alcalino}

Recentemente, combinados pré-tratamentos têm sido considerados um meio promissor para superar o desafio de aumentar a eficiência da produção de açúcares a partir da degradação de polissacarídeos, visto que essas combinações visam à exposição da molécula de celulose para facilitar a fase seguinte que é a hidrólise. Diversas combinações têm sido realizadas, por exemplo, combinação dos prétratamentos alcalino e líquido iônico, ácido e explosão a vapor, $\mathrm{CO}_{2}$ supercrítico e vapor, organosolv e biológico, ácido e alcalino, entre outros, sendo esse último o mais comumente empregado nas pesquisas atuais.

O pré-tratamento ácido poderia deslignificar e aumentar a área superficial da fibra de celulose, entretanto, um único estágio de pré-tratamento com ácido acético, por exemplo, necessita de uma concentração em torno de 50\%, baseada na quantidade inicial de material seco, então, para superar esse desafio, um pré-tratamento combinado poderia ser aplicado para parcialmente, também remover parte da lignina em um único estágio (MOOD et al.,2013). 
Mendes et al. (2012) avaliaram sete diferentes estratégias de pré-tratamento para a biomassa lignocelulósica Brachiaria brizantha cv. Marandu e o pré-tratamento ácido $\left(\mathrm{H}_{2} \mathrm{SO}_{4}\right)$ seguido de básico $(\mathrm{NaOH})$, ambos a $4 \%$, apresentou os melhores resultados dentre os avaliados, este resultou em um material com 92,36\% de celulose, sua hidrólise enzimática apresentou conversão de 94,62\% da celulose em glicose.

Filho (2014) ao estudar a produção de etanol celulósico a partir da palma forrageira, realizou um pré-tratamento ácido $\left(\mathrm{H}_{2} \mathrm{SO}_{4}\right)$ seguido de básico $(\mathrm{NaOH})$, ambos a $0,5 \%$, e obteve uma recuperação de celulose em torno de 32,31\% e um percentual de 24,19\% de deslignificação do material lignocelulósico.

\section{Considerações Finais}

Observa-se então que as fibras lignocelulósicas são matérias-primas promissoras para a produção de etanol de segunda geração visto que apresentam um percentual significativo de hemicelulose e celulose, que são carboidratos que podem ser degradados em açúcares fermentescíveis que com a ação de leveduras podem ser metabolizados bioquimicamente a etanol.

\section{Lignocellulosic Fibers Potential for the Second Generation Ethanol Production}

Albstract: The production of chemicals and fuels from lignocellulosic materials has been a particular interest of researchers due to the negative impact of fossil fuel into the environment, but also increasing concern with geopolitical issues of oil worldwide. Current research shows as an alternative technology the second-generation ethanol from lignocellulosic materials. The second-generation ethanol corresponds to ethanol production, however, using as raw lignocellulosic material. The lignocellulosic materials are composed primarily of cellulose, hemicellulose and lignin. These three components are essential to the production of this biofuel, since the breakdown of these molecules will produce sugars which can with the aid of yeasts biochemically be converted into ethanol and other chemicals. The region's semi-arid northeast, between eight northeastern states: Alagoas, Bahia, Ceará, Paraíba, Pernambuco, Piauí, Rio Grande do Norte and Sergipe has promising materials for the production of these biofuels such as bagasse and straw sugarcane, sisal fiber, cactus pear and cashew bagasse. Besides the choice of suitable raw materials for the production of ethanol 2G, the steps involved in this process, pre-treatment, hydrolysis, fermentation and distillation will depend mainly on the chosen culture and its physicochemical composition. Thus, the main objective of this work is to show the potential of lignocellulosic fibers for second-generation ethanol and study three main types of pretreatment therefor: acid, basic and acid-base combination.

Keywords: Pretreatment; Lignocellulosic Material; Fermentable Sugars; Northeast

\section{Referências bibliográficas}

ARANTES, Valdeir; SADDLER, Jack N. Access to cellulose limits the efficiency of enzymatic hydrolysis: the role of amorphogenesis. Biotechnol Biofuels, [s.l.], v. 3, n. 1, p.4-15, 2010.

CAMPOS, L.M.A.; PONTES, L.A.M.; CARVALHO, L.S.; CHEMMÉS, C.S.; GARRIDO, C.V.S.; SILVA, F.C.; SANTOS, J.M.G.M. ; LEAL, S.C.S. ; MERCANDELLI, S.S. ; SILVA, V.L. Avaliação de Pré-tratamento do Bagaço de Cana-de-Açúcar com Ácido Diluído para Produção de Etanol de Segunda Geração. 53 Congresso Brasileiro de Química. Rio de Janeiro/RJ, 2013.

CARDOSO, V. M. Aplicação da radiação de feixe de elétrons como pré-tratamento do bagaço da cana-de-açúcar para hidrólise enzimática da celulose. Dissertação (Ciências na Área de Tecnologia Nuclear - Aplicações), Instituto de Pesquisas Energéticas e Nucleares - IPEN, São Paulo, 2008. 
CASTRO, A.M, JUNIOR, N.P. Produção, propriedades e aplicação de celulases na hidrólise de resíduos agroindustriais. Quim. Nova, Vol. 33, No. 1, 181-188, 2010.

CHOVAU, S.; DEGRAUWE, D.; VAN DER BRUGGEN, B. Critical analysis of techno-economic estimates for the production cost of lignocellulosic bio-ethanol. Renewable and Sustainable Energy Reviews. pp. 307-321. 2013.

EPE - Empresa de Pesquisa Energética. National Energy Balance. Diponível em: https://ben.epe.gov.br/.

FERREIRA, LL. Flexibilidade na utilização de diesel ou biodiesel, uma abordagem utilizando a teoria de opções reais. Dissertação de Mestrado. Rio de JaneiroRJ: Fundação Getulio Vargas; 2007.

FILHO, P.F.S., Palma forrageira (Opuntia fícus indica e Nopalea cochelillifera como matériaprima para a produção de etanol celulósico e enzimas celulóticas. Dissertação de Mestrado. Universidade Federal do Rio Grande do Norte, Natal, 2014

GOMEZ, E. O., SOUZA, R. T. G., ROCHA, G.J.M., ALMEIDA, E., CORTEZ, L. A.B. Avaliação de resíduos lignocelulósicos para a produção de etanol EG. Revista Analytica, v.48, p. 180, 2010.

IEA — International Energy Agency. World Energy Outlook. IEA, Paris. 2013

KUMAR, P., BARRET, D.M.,DELWICHE; M. L. J. E., STROEVE, P. Methods for pretreatment of lignocellulosic biomass for efficient hydrolysis and biofuel production. Ind. Engineering Chemical Research, v. 48, p. 3713-3729,2009.

LEITE, R.C.C.; LEAL, M.R.L. V; CORTEZ, L.A.B.; GRIFFIN, W.M.;SCANDIFFIO,M.I. G. Can Brazil replace 5\% of the 2025 gasoline world demand with ethanol? Energy Applied, 34 (5), pp. 655661, 2009.

LI, X.; KIM, TH.; Nghiem, NP. Bioethanol production from corn stover using aqueous ammonia pretreatment and two-phase simultaneous saccharification and fermentation (TPSSF). Bioresource Technology.p. 5910-5916, 2010

LIMA, A. O. S.; RODRIGUES, A. L. Sacarificação de resíduos celulósicos com bactérias recombinantes como estratégia para redução do efeito estufa. Revista de ciências ambientais, v. 1, n. 2, p. 5-18, 2007.

MELO, L. F. L.; Substâncias químicas em efluentes da extração de nanocristais de celulose. Trabalho de Conclusão de Curso (Bacharelado em Química), Universidade Federal do Ceará, Fortaleza, 2010.

MENDES, T. D.; PACHECO, T. F.; CARVALHO, F.B.P.; NAKAI, D. K.; RODRIGUES, D. S.; MACHADO, C. M. M., AYRES M. Avaliação de diferentes pré-tratamentos para desconstrução da biomassa lignocelulósica. Anais do XIX Congresso Brasileiro de Engenharia Química. Búzios - RJ, 2012.

MOOD, S. H.; GOLFESHAN, A. H.; TABATABAEI M.; JOUZANI, G. S.; NAJAFI, G.H; GHOLAMI, M.;ARDJMAND, M. Lignocellulosic biomass to bioethanol, a comprehensive review with a focus on pretreatment. Renewable and Sustainable Energy Reviews. V. 27, p.77-93, 2013.

MORAIS, S.A.L.; NASCIMENTO, E.A.; MELO, D.C. Chemical analysis of Pinusoocarpa wood PART I - Quantification of macromolecular components and volatile extractives. Revista Árvore, v. 29, n. 3, p. 461-470, 2005. 
NASCIMENTO, V.M. Pré-tratamento alcalino do bagaço de cana-de-açúcar para produção de etanol e obtenção de xilooligômeros. Dissertação de Mestrado. Universidade Federal de São Carlos. São Paulo. 126 p, 2011.

OLIVEIRA, F.M.V., Avaliação de diferentes pré-tratamentos e deslignificação alcalina na sacarificação da celulose de palha de cana. Dissertação de Mestrado. Escola de Engenharia de Lorena da Universidade de São Paulo. 98 p. 2010

PEREIRA Jr., N.; COUTO, M.A.P.G.; SANTA, L.M.M. Biomass of lignocellulosic composition for fuel ethanol production and the context of biorefinery. In Series on Biotechnology, Ed. Amiga Digital UFRJ, Rio de Janeiro, v.2, 45 p, 2008.

PINHEIRO, F.G.C; COSTA, A.G. ; MORAIS, J.P.S. ; SANTOS, A.B. ; SANTAELLA, S.T.; LEITÃO, R.C. Pré-tratamento termoquímico do bagaço da cana-de-açúcar para a produção de açúcares fermentescíveis. II Simpósio Internacional sobre Gerenciamento de Resíduos Agropecuários e Agroindustriais, 2011.

RODRIGUES, J.A.R. Do engenho à biorrefinaria: A usina de açúcar como empreendimento industrial para a geração de produtos bioquímicos e biocombustíveis. Instituto de Química, Universidade de Campinas, Campinas - SP, Brasil, 2010

RODRIGUES, L.D. A cana-de-açúcar como matéria-prima para a produção de biocombustíveis: impactos ambientais e o zoneamento agroecológico como ferramenta para mitigação. Trabalho de conclusão de curso em análise ambiental. Universidade Federal de Juiz de Fora. 59 p. 2011.

SILVEIRA, M. S. Aproveitamento das cascas de coco verde para produção de briquete em Salvador - BA. Dissertação (Mestre em Gerenciamento e Tecnologias Ambientais no Processo Produtivo). Universidade Federal da Bahia, Salvador, 2008.

TAHERZADEH, M.J. \& KARIMI, K. Pretreatment of Lignocellulosic Wastes to Improve Ethanol and Biogas Production: A Review. International Journal of Molecular Sciences, 9, pp.1621-1651. 2008.

TOLMASQUIM, M.T. Perspectivas e planejamento do setor energético no Brasil. Estudos Avançados;26(74):247-60, 2012. 